\title{
Hierarchical Control Design of a UAV Helicopter
}

\author{
Ali Karimoddini, Guowei Cai, Ben M. Chen, Hai Lin, Tong H. Lee \\ Graduate School for Integrative Sciences and Engineering (NGS) and Department of \\ Electrical and Computer Engineering, National University of Singapore \\ Singapore
}

\section{Introduction}

Nowadays, control design of Unmanned Aerial Vehicles (UAVs) has emerged as an attractive research area, due to the wide range of UAV applications in various military and civilian areas such as terrain and utility inspections, coordinated surveillance, search and rescue missions, disaster monitoring, rapid emergency response, aerial mapping, traffic monitoring, and reconnaissance missions (see, e.g., (Metni et al., 2007), (Kuroki et al., 2010 ), (Campbell \& Campbell, 2010 )). They can also be used as complex test-bed dynamic systems for implementation and verification of the control schemes for different research purposes (Kim \& Sukkarieh, 2007), (Saripalli et al., 2003), (Bortoff, 1999). Several research groups are involved in the modeling and control of UAVs (Bortoff, 1999), (Gavrilets et al., 2000), (Cai et al., 2006). The control methods such as the neural network approach (Enns \& Si, 2003), the differential geometry method (Isidori et al., 2003), feedback control with decoupling approach (Peng et al., 2009), and the model predictive approach (Shim et al., 2003) have been applied for the flight control of the UAV helicopters. In this chapter, however, we have used an analytical approach to design and analyze the whole system including the inner-loop and the outer-loop controllers for a small-scale UAV helicopter. Here, in the proposed hierarchical structure, the inner-loop is responsible for the internal stabilization of the UAV in the hovering state and control of the linear velocities and heading angle angular velocity whereas the outer-loop is used to drive the system, which is already stabilized by the inner-loop, to follow a desired path while keeping the system close to the hovering state. This hierarchical strategy is an intuitive way of controlling such a complex system. However, there is another reason that compels us to employ such a control structure. Indeed, the UAV model cannot be fully linearized, since, in practice, we cannot expect the heading angle of the UAV to be restricted to a small range of variation as depending on the mission, the heading of the UAV could be in any direction. This will impose some kind of nonlinearity on the system, which can be modeled by a simple transformation. To handle this semi-linearized model of the UAV, we can separate the linear and nonlinear parts, and then control the linear part in the inner-loop and the nonlinear part in the outer-loop.

In this hierarchy, for the inner-loop, we have used an $H_{\infty}$ controller to both stabilize the system and suboptimally achieve the desired performance of the UAV attitude control. Assuming that the inner-loop has already been stabilized by an $H_{\infty}$ controller, a proportional feedback controller combined with a nonlinear compensator block have been used in the outer-loop to bring the UAV into the desired position with desired heading angle. 
Although designing a proportional feedback controller for SISO systems is straightforward, the situation for MIMO systems is different. This is because, in MIMO systems, it is not easy to use the popular tools, such as the Nyquist stability theorem or the root-locus approach, that are well-established for the SISO systems. The current approaches employed for MIMO systems are rather complicated and are mostly extensions of the existing results for SISO systems (Wang et al., 2007). In this chapter, we propose a design method of a decentralized P-controller for MIMO systems that, although conservative, can be effectively used in practical problems, particularly for the case that the system is close to a decoupled system. The approach is an extension of the Nyquist theorem to MIMO systems, and its application to the NUS UAV system provides a successful flight controlled system.

The test-bed is Helion (Fig. 1), the first developed UAV helicopter in our NUS UAV research group (Peng et al., 2007). In (Cai et al., 2008a), a systematic procedure for the construction of a small-scale UAV helicopter is described, and in (Cai et al., 2005), the hardware parts of the NUS UAV, including both the avionic system and the ground station, are illustrated in detail.

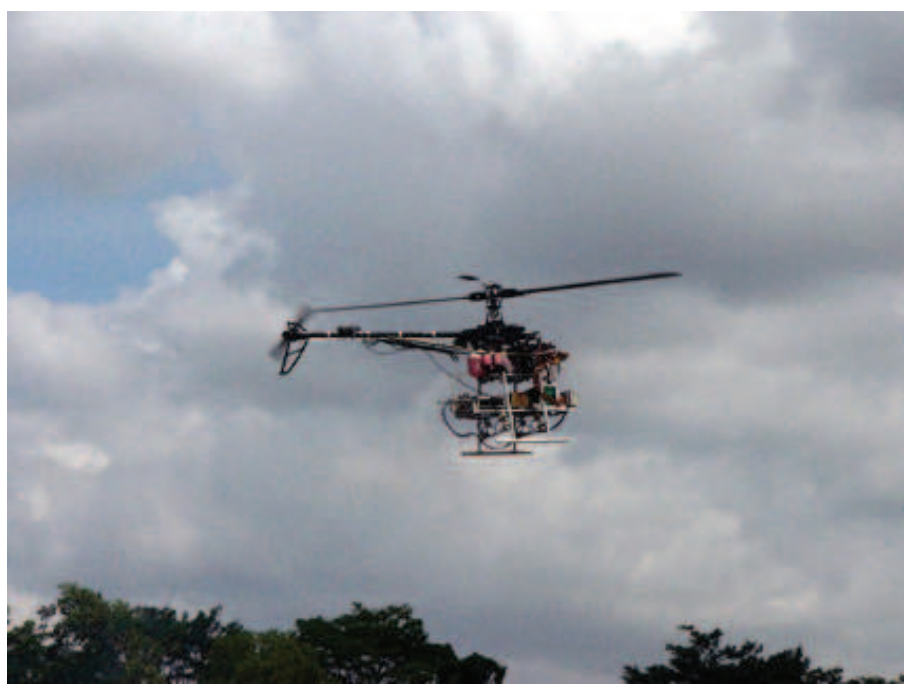

Fig. 1. The NUS UAV helicopter.

The remaining parts of this chapter are organized as follows. In Section II, the model and the structure of the NUS UAV is described. The UAV model consists of two decoupled subsystems. In Section III, a hierarchical controller, including an inner-loop and an outer-loop controller, is designed for both subsystems. Actual flight tests are presented in Section IV, and the chapter is concluded in Section V. For the convenience of the reader, a nomenclature part is provided at the end of this chapter.

\section{Modeling and structure of the UAV helicopter}

A typical UAV helicopter consists of several parts: physical parts such as engine and fuselage; ground station to monitor the flight situation and collect realtime flight data, and the avionic system to implement the control strategy to have an autonomous flight control. Among these elements, the avionic part is in the center of our interest in this chapter and we will focus on the control structure which is embedded in the airborne computer system. Here, the avionic 
system consists of an airborne computer system which can be extended modularly by some extension boards such as $\mathrm{A} \backslash \mathrm{D}$ card, DC-DC convertor card, and serial communication board. In addition, the avionic system has been equipped with some analog and digital sensors to collect the information of the current state of the UAV. The major sensor used in the avionic system, is the NAV-IMU sensor. The IMU sensor provides three axis velocities, acceleration and angular rates in the body frame, as well as longitude, latitude, relative height and heading, pitch and roll angles. Moreover, the avionic system has a fuel level sensor as well as a magnetic RPM sensor to measure the speed of the rotor. Furthermore, it comprises five servo actuators that could manipulate the helicopter to move forward and backward, up and down, to turn left and right, to regulate the nose angle and finally to control the spinning speed of the rotor. All of these servos are controlled by a servo board as a local controller. In addition, the servo board gives the ability of driving the servo system into either the manual mode or the automatic mode. In the manual mode, a pilot can drive the helicopter by a radio controller which is useful in the emergency situations; however, in the automatic mode the helicopter is under the control of the computer system and all control signals are generated by the avionic system and the computer board, autonomously.

Using some basic physical principles, we can obtain a general nonlinear UAV model. These principles will result in several equations that represent the effects of different factors such as gravity, the main rotor, and tail rotor forces and moments. The model equations will be obtained in two coordinate systems: the body frame and the ground frame. The body frame is centered at the center of gravity of the UAV, and the ground frame is an NED (North - East - Down) coordinate system (Stevens \& Lewis, 1992) with a fixed origin at the starting point of the UAV flight. Clearly, the UAV dynamic equations should be derived in the body frame, while the position of the UAV is considered in the ground frame.

Neglecting the gyroscopic effect of the engine-driven train, the equations of the helicopter motion in the body frame are obtained as follows:

$$
\begin{gathered}
\dot{\vec{V}}_{b}=-\vec{\omega}_{b} \times \vec{V}_{b}+B_{b} \vec{g}+m^{-1} \vec{F} \\
\dot{\vec{\omega}}_{b}=-J^{-1} \omega_{b} \times J \vec{\omega}_{b}+J^{-1} \vec{M}
\end{gathered}
$$

where in these equations, $\times$ denotes the cross product of the vectors, and the concatenation of two matrices or vectors represents the normal matrix multiplication. Moreover, $\vec{F}$ and $\vec{M}$ are the resultant force and moment in the body frame, including those generated from the main rotor, the tail rotor and the fuselage. Other symbols' definition can be found in the nomenclature part provided at the end of this chapter.

The Euler angles that show the orientation of the body frame relative to the ground frame are as follows:

$$
\left[\begin{array}{c}
\dot{\phi} \\
\dot{\theta} \\
\dot{\psi}
\end{array}\right]=\left[\begin{array}{ccc}
1 & \tan \theta \sin \phi & \tan \theta \cos \phi \\
0 & \cos \phi & -\sin \phi \\
0 & \frac{\sin \phi}{\cos \theta} & \frac{\cos \phi}{\cos \theta}
\end{array}\right] \dot{\vec{\omega}}_{b}
$$

where $(\phi \theta \psi)^{\prime}$ is a vector that contains the Euler angles to describe the attitude of the helicopter with respect to the NED frame.

The relation between the UAV position in the ground frame and the UAV velocity in the body frame is:

$$
\dot{\vec{P}}_{g}=\dot{B}_{b} \vec{V}_{b}
$$


where $B_{b}$ is the transformation matrix from the ground frame to the body frame, which has the following form:

$$
B_{b}=\left[\begin{array}{ccc}
\cos \theta \cos \psi & \cos \theta \sin \psi & -\sin \theta \\
-\cos \phi \sin \psi+\sin \phi \sin \theta \cos \psi & \cos \phi \cos \psi+\sin \phi \sin \theta \sin \psi & \sin \phi \cos \theta \\
\sin \phi \sin \psi+\cos \phi \sin \theta \cos \psi & -\sin \phi \cos \psi+\cos \phi \sin \theta \cos \psi & \cos \phi \cos \theta
\end{array}\right]
$$

The details of this UAV model are described in (Peng et al., 2007). From the above model description, it can be seen that the UAV model is nonlinear. Furthermore, the main problem encountered in the modeling of our UAV is that the process of buying a radio-control helicopter from the market and upgrading it to an autonomous flying vehicle leaves us with many unknown parameters and aerodynamic data. Therefore, for practical reasons, we need to linearize the UAV model and then, identify the resulting linearized model through the recorded in-flight data. The in-flight data can be collected through the manual mode and by injecting perturbed input signals to the flying helicopter. We have obtained a linearized model at the hovering state as it has been presented in (Cai et al., 2006). By hovering, we mean that $\vec{V}_{0}=0, \vec{\omega}_{0}=0, \theta_{0}=0, \phi_{0}=0$. The obtained linearized model is as follows:

$$
\dot{x}=A x+B u+E w
$$

where the state variable $x$ includes 11 variables as $x=\left[V_{z_{b}}(\mathrm{~m} / \mathrm{s}) \omega_{z_{b}}(\mathrm{rad} / \mathrm{s}) w_{z f}(\mathrm{rad} / \mathrm{s})\right.$ $\left.V_{x_{b}}(\mathrm{~m} / \mathrm{s}) V_{y_{b}}(\mathrm{~m} / \mathrm{s}) \omega_{x_{b}}(\mathrm{rad} / \mathrm{s}) \omega_{y_{b}}(\mathrm{rad} / \mathrm{s}) \phi(\mathrm{rad}) \theta(\mathrm{rad}) \tilde{a}_{s}(\mathrm{rad}) \tilde{b}_{s}(\mathrm{rad})\right]^{\prime}$. These parameters are shown in Fig. 2. $w_{z f}$ is the yaw rate feedback, which is related to $\delta_{\text {pedal }}$ by a first-order differential equation (Cai et al., 2008b).

Furthermore, the control input $u$ includes commands to the servos embedded for the control of the helicopter blades as $u=\left[\delta_{\text {col }}(\mathrm{rad}) \delta_{\text {pedal }}(\mathrm{rad}) \delta_{\text {roll }}(\mathrm{rad}) \delta_{\text {pitch }}(\mathrm{rad})\right]^{\prime}$.

Matrices $A, B$, and $E$ are obtained as follows:

$$
A=\left[\begin{array}{cc}
A_{1} & 0_{3 \times 8} \\
0_{8 \times 3} & A_{2}
\end{array}\right], B=\left[\begin{array}{cc}
B_{1} & 0_{3 \times 2} \\
0_{8 \times 2} & B_{2}
\end{array}\right], E=\left[\begin{array}{cc}
E_{1} & 0_{3 \times 2} \\
0_{8 \times 1} & E_{2}
\end{array}\right]
$$

$$
\begin{aligned}
& A_{1}=\left[\begin{array}{ccc}
-0.6821 & -0.1070 & 0 \\
-0.1446 & -5.5561 & -36.6740 \\
0 & 2.7492 & -11.1120
\end{array}\right], B_{1}=\left[\begin{array}{cc}
15.6491 & 0 \\
1.6349 & -58.4053 \\
0 & 0
\end{array}\right] \text {, } \\
& E_{1}=\left[\begin{array}{c}
-0.5995 \\
-1.3832 \\
0
\end{array}\right], B_{2}=\left[\begin{array}{cc}
0 & 0 \\
0 & 0 \\
0 & 0 \\
0 & 0 \\
0 & 0 \\
0 & 0 \\
0.0496 & 2.6224 \\
2.4928 & 0.1740
\end{array}\right], E_{2}=\left[\begin{array}{cc}
-0.1778 & 0 \\
0 & -0.3104 \\
-0.3326 & -0.2051 \\
0.0802 & -0.2940 \\
0 & 0 \\
0 & 0 \\
0 & 0 \\
0 & 0
\end{array}\right] \text {, } \\
& A_{2}=\left[\begin{array}{cccccccc}
-0.1778 & 0 & 0 & 0 & 0 & -9.7807 & -9.7808 & 0 \\
0 & -0.3104 & 0 & 0 & 9.7807 & 0 & 0 & 9.7807 \\
-0.3326 & -0.5353 & 0 & 0 & 0 & 0 & 75.7640 & 343.86 \\
-0.1903 & -0.2940 & 0 & 0 & 0 & 0 & 172.620 & -59.958 \\
0 & 0 & 1 & 0 & 0 & 0 & 0 & 0 \\
0 & 0 & 0 & 1 & 0 & 0 & 0 & 0 \\
0 & 0 & 0 & -1 & 0 & 0 & -8.1222 & 4.6535 \\
0 & 0 & -1 & 0 & 0 & 0 & -0.0921 & -8.1222
\end{array}\right] .
\end{aligned}
$$

Remark 1. In the linearized model described by (6), the saturation level for the servos is $\left|\delta_{\star_{\max }}\right|=0.5$. We need to provide a control law such that the resulting control signals always remain within the linear unsaturated range. 


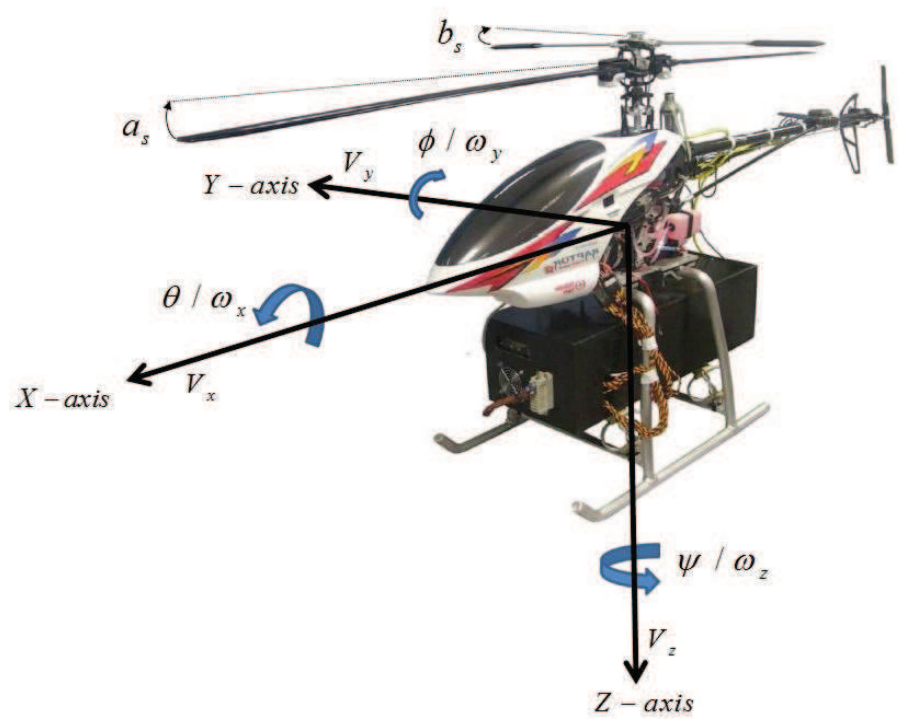

Fig. 2. Helicopter states in the body frame.

Although (6) describes the relation between the control input and the state variable $x$, it still does not describe the whole dynamics of the system, and particularly, $\vec{P}$ and $\varphi$ are not reflected in the model. Thus, considering (2) and (4), a more complete model containing $\vec{P}$ and $\varphi$ is as follows:

$$
\left\{\begin{array}{l}
\dot{x}=A x+B u+E w \\
\dot{\psi}=\omega_{z_{b}} \\
\dot{\vec{P}}_{g}=\dot{B}_{b} \vec{V}_{b}
\end{array}\right.
$$

Remark 2. Matrix $B_{b}$ includes some time-dependent terms. Therefore, matrix $B_{b}$ can not be considered as a constant term and it is not simple to integrate both sides of (7) in order to obtain the position in the ground frame. This is due to the fact that the body frame is a moving coordinate system. Hence, to obtain the displacement, it is necessary to first obtain the velocities in a fixed coordinate system such as the ground frame. Then, the displacement can be calculated by integrating of the velocity vector in the fixed coordinate system.

The presence of nonlinear terms of $B_{b}$, in the third equation of (7), makes it difficult to design a controller for the system; however, we can further simplify the model. Indeed, matrix $B_{b}$ in (5), which introduces some nonlinear terms to the model, can be linearized at the hovering state. In practice, the heading angle of the helicopter can take any arbitrary value; however, the roll and pitch angles are usually kept close to the hovering condition. Therefore, linearizing matrix $B_{b}$ at the hovering state will result in:

$$
B_{b}=\left[\begin{array}{ccc}
\cos \psi & \sin \psi & 0 \\
-\sin \psi & \cos \psi & 0 \\
0 & 0 & 1
\end{array}\right]=\left[\begin{array}{cc}
R & \mathbf{0}_{\mathbf{2} \times \mathbf{1}} \\
\mathbf{0}_{\mathbf{1} \times \mathbf{2}} & 1
\end{array}\right]
$$

The physical interpretation is that by keeping $\vec{\theta}$ and $\vec{\phi}$ close to zero, the Euler rotation in a three-dimensional space will be converted into a simple rotation in a two-dimensional space 
with respect to $\psi$. In this case, the rotation matrix is:

$$
R=\left[\begin{array}{cc}
\cos \psi & \sin \psi \\
-\sin \psi & \cos \psi
\end{array}\right]
$$

In the following section, as we design the outer-loop controller, it will be shown that this new formulation of $B_{b}$ helps us to keep the system decoupled, even after using the outer-loop controller.

The semi-linearized UAV model, presented in (7) can be controlled in separate parts: the linear part in the inner-loop and the nonlinear part in the outer-loop, as described in the following section.

\section{Controller design}

We use a hierarchical approach to design a controller for the UAV (Fig. 3). In this framework, the system is stabilized in the inner-loop, and then it is driven to track a desired trajectory in the outer-loop. Besides this rational strategy, there is another reason that compels us to select this particular architecture. As mentioned previously, we need to derive the model equations in two coordinate systems: the velocities and accelerations should be obtained in the body coordinate system as a moving frame, whereas the displacement must be derived by the integration of the velocities in a fixed frame. The velocity transformation from the body frame to the ground frame, modeled by matrix $B_{b}$, imposes some kind of nonlinearity as described in (7). This nonlinearity can be handled in the outer-loop. Using this control strategy, we have separated the nonlinear term from the linear part and put it in the outer-loop.

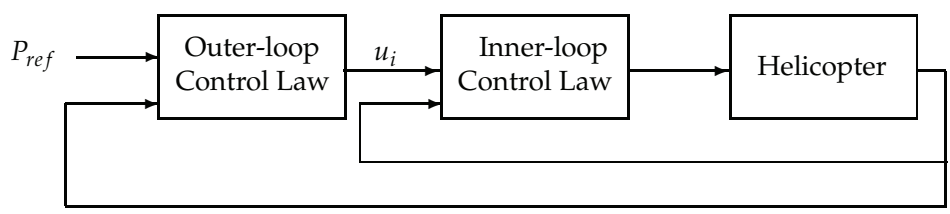

Fig. 3. Schematic diagram of the flight control system.

In this control architecture, the references for the inner-loop controller, $u_{i}$, are the linear velocities $\left(V_{x_{b}}, V_{y_{b}}\right.$, and $\left.V_{z_{b}}\right)$ and the yaw rate, $\omega_{z_{b}}$, which should all be provided by the outer-loop. The outer-loop, however, is responsible for the control of the position and heading angle of the UAV and will guide the UAV to follow a desired trajectory. Therefore, the references for the outer-loop are the position $\left(X_{r}, Y_{r}, Z_{r}\right)$ and the yaw angle $\psi_{r}$. In other words, the UAV will follow the generated path by the position and the yaw angle control in the outer-loop, and the linear velocity and the angular rate control in the inner-loop.

looking at matrices $A, B$, and $E$ in (6), we can see that, the system has been already decoupled into two independent parts. Therefore, (6) can be rewritten into two separate subsystems as follows:

$$
\begin{aligned}
& \dot{x}_{1}=A_{1} x_{1}+B_{1} u_{1}+E_{1} w \\
& \dot{x}_{2}=A_{2} x_{2}+B_{2} u_{2}+E_{2} w
\end{aligned}
$$

where $x_{1}=\left[V_{z_{b}}(\mathrm{~m} / \mathrm{s}) \omega_{z_{b}}(\mathrm{rad} / \mathrm{s}) w_{z f}(\mathrm{rad} / \mathrm{s})\right]^{\prime}, u_{1}=\left[\delta_{\text {col }} \delta_{\text {pedal }}\right]^{\prime}, x_{2}=\left[V_{x_{b}}(\mathrm{~m} / \mathrm{s}) V_{y_{b}}(\mathrm{~m} / \mathrm{s})\right.$ $\left.\omega_{x_{b}}(\mathrm{rad} / \mathrm{s}) \omega_{y_{b}}(\mathrm{rad} / \mathrm{s}) \phi(\mathrm{rad}) \theta(\mathrm{rad}) \tilde{a}_{s}(\mathrm{rad}) \tilde{b}_{s}(\mathrm{rad})\right]^{\prime}$, and $u_{2}=\left[\delta_{\text {roll }}(\mathrm{rad}) \delta_{\text {pitch }}(\mathrm{rad})\right]^{\prime}$. 
Considering (7), (8), (10), and (11), the above-mentioned hierarchical control strategy can be implemented for the decoupled model of our UAV, as shown in Fig. 4 and Fig. 5. In these figures, subscripts $g, b$, and $r$ stand for ground frame, body frame, and reference, respectively. Moreover, matrices $C_{1}$ and $C_{2}$ are:

$$
C_{1}=\left[\begin{array}{lll}
1 & 0 & 0 \\
0 & 1 & 0
\end{array}\right], C_{2}=\left[\begin{array}{llllllll}
1 & 0 & 0 & 0 & 0 & 0 & 0 & 0 \\
0 & 1 & 0 & 0 & 0 & 0 & 0 & 0
\end{array}\right]
$$

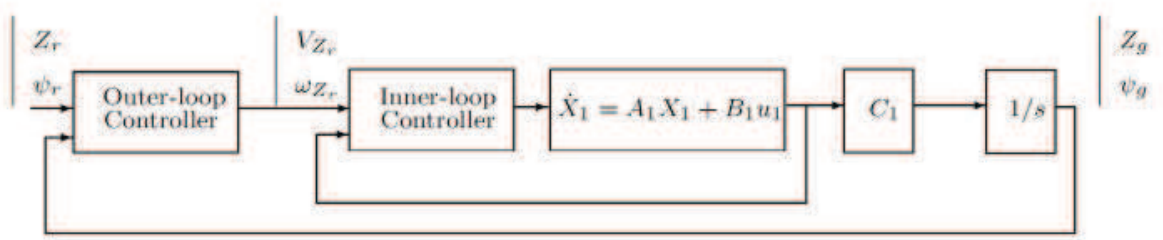

Fig. 4. Control schematic for Subsystem 1.

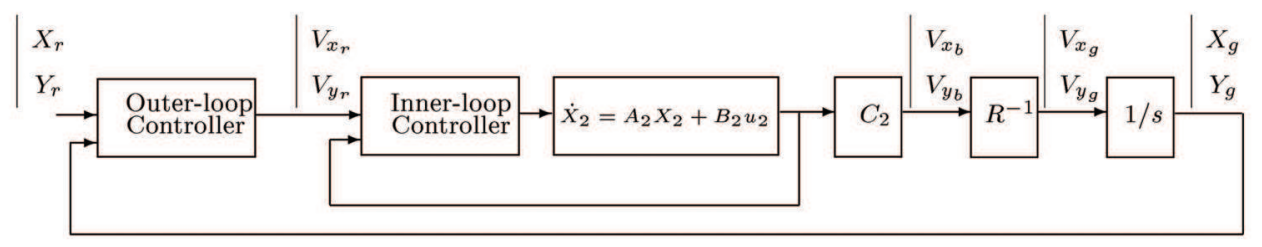

Fig. 5. Control schematic for Subsystem 2.

Due to the special structure of the linearized form of $B_{b}$, Subsystem 1 is a fully linearized model. However, in the outer-loop of Subsystem 2, the term $R^{-1}$ appears as a nonlinear element, and thus Subsystem 2 is more complicated than Subsystem 1. In the following parts, we will describe the control design for both subsystems.

\subsection{Controller for subsystem 1}

Subsystem 1 is a fully linearized model so that linear design tools can be applied to the system. We will use the $H_{\infty}$ control design technique for the inner-loop and a P-controller for the outer-loop.

\subsubsection{Inner-loop controller}

Using an $H_{\infty}$ controller for the inner-loop, both robust stability and proper performance of the system can be achieved simultaneously. To design the $H_{\infty}$ controller, using notation analogous with (Chen, 2000), we define the measurement output simply as the state feedback in the form of $y_{1}=C_{11} x$ with $C_{11}=I$. Since our primary task is to design a control law to internally stabilize the system, and to achieve a good response of the state variables that are directly related and linked to the outer-loop, while considering the constraints on the inputs and some state variables, we define the controlled output $h_{1}$ in the form of $h_{1}=C_{12} x+D_{12} u$, where

$$
C_{12}=\left[\begin{array}{ccc}
3.1623 & 0_{2 \times 3} & 0 \\
0 & 3.1623 & 0 \\
0 & 0 & 1.7321
\end{array}\right], D_{12}=\left[\begin{array}{cc}
44.7214 & 0 \\
0 & 28.2843 \\
03 \times 2
\end{array}\right]
$$


The nonzero entries of $C_{12}$ and $D_{12}$ are used for tuning the controller, and here, are determined experimentally to achieve the desired performance. Meanwhile, the $H_{\infty}$ design guarantees internal stability and robustness of the system. Indeed, $H_{\infty}$ control design minimizes the effect of the wind gust disturbance, i.e., minimizes the $H_{\infty}$ norm of the closed-loop transfer function from the disturbance $w$ to the controlled output $h_{1}$, denoted by $T_{1}$. The $H_{\infty}$ norm of the transfer function $T_{1}$ is defined as follows:

$$
\left\|T_{1}\right\|_{\infty}=\sup _{0 \leq \omega<\infty} \sigma_{\max }\left[T_{1}(j \omega)\right]
$$

where $\sigma_{\max }[$.$] denotes the maximum singular value of the matrix.$

It should be highlighted that the $H_{\infty}$ norm is the worst case gain in $T_{1}(s)$. Therefore, minimization of the $H_{\infty}$ norm of $T_{1}$ is equivalent to the minimization of the disturbance effect from the disturbance $w$ to the controlled output $h_{1}$ in the worst case situation. Having the matrix $C_{12}$ and $D_{12}$, one can find the $\gamma_{\infty}^{*}$ which is the optimal $H_{\infty}$ performance for the closed-loop system from the disturbance input $w$ to the controlled output $h_{1}$ over all the possible controllers that internally stabilize the system. As practically, $\gamma_{\infty}^{*}$ is not achievable, we will try to reach $\gamma_{\infty}$ which is slightly larger than $\gamma_{\infty}^{*}$.

With this choice of the control parameters, $D_{11}$ and $D_{12}$ are full rank and the quadruples $\left(A_{1}, B_{1}, C_{12}, D_{12}\right)$ and $\left(A_{1}, E_{1}, C_{11}, D_{11}\right)$ are left invertible and are free of invariant zeros. Therefore, we have a so-called regular problem, for which we can use well-established $H_{\infty}$ control theory (Chen, 2000). The resulting closed loop system suboptimality minimizes the $H_{\infty}$ norm of the transfer function from the disturbance $w$ to the controlled output $h_{1}$. To design this controller we consider the control law in the following form:

$$
u_{1}=F_{1} x_{1}+G_{1} r_{1}
$$

where $r_{1}=\left(V_{z_{r}}, \omega_{z_{r}}\right)^{\prime}$ is the reference signal generated by the outer-loop controller, $G_{1}=$ $-\left(C_{1}\left(A_{1}+B_{1} F_{1}\right)^{-1} B_{1}\right)^{-1}$ is the feedforward gain, and $F_{1}$ is the $H_{\infty}$ control gain that can be achieved as follows:

$$
\left.F_{1}=-\left(D_{12}^{\prime} D_{12}\right)^{-1}\left(D_{12}^{\prime} C_{12}+B_{1}^{\prime} P_{1}\right)\right)
$$

where matrix $P_{1}$ is the positive semi-definite solution of the following $H_{\infty}$ algebraic Riccati equation:

$$
\begin{array}{r}
A_{1}^{\prime} P_{1}+P_{1} A_{1}+C_{12}^{\prime} C_{12}+P_{1} E_{1} E_{1}^{\prime} P_{1} / \gamma^{2}- \\
\left(P_{1} B_{1}+C_{12}^{\prime} D_{12}\right)\left(D_{12}^{\prime} D_{12}\right)^{-1}\left(D_{12}^{\prime} C_{12}+B_{1}^{\prime} P_{1}\right)=0
\end{array}
$$

For this system and these control parameters values, the value of $\gamma_{\infty}^{*}$ is 1.4516 . Hence, we select $\gamma_{\infty}=1.4616$. Therefore, matrices $F_{1}$ and $G_{1}$ are obtained as follows:

$$
F_{1}=\left[\begin{array}{ccc}
-0.0935 & -0.0005 & 0.0027 \\
0.0008 & 0.0364 & -0.0481
\end{array}\right], G_{1}=\left[\begin{array}{cc}
0.1371 & 0.0066 \\
-0.0020 & -0.2748
\end{array}\right]
$$

To evaluate the controller performance and its effect on the disturbance attenuation, we simulated the closed loop system with an initial state of $x_{1}\left(\begin{array}{l}0 \\ 0\end{array}=\left[\begin{array}{lll}1.5 & 0 & 0\end{array}\right]^{\prime}\right.$, and also we injected wind gust disturbance for $20 \mathrm{sec}$ (Fig. 6). The injected disturbance has a maximum amplitude of $3 \mathrm{~m} / \mathrm{s}$ along the $\mathrm{z}$ axis (the other directions do not affect the dynamics of Subsystem 1). The controlled system reaches the steady hovering state after $3.5 \mathrm{sec}$, and the disturbance effect is reduced to less than $0.25 \%$. The control inputs are within the unsaturated region. 

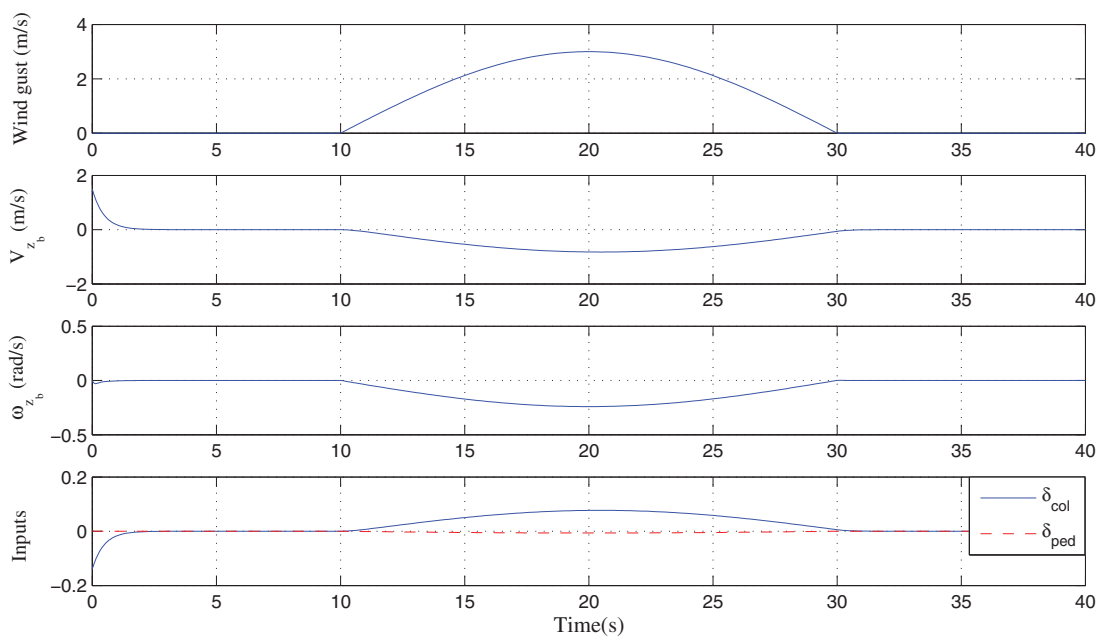

Fig. 6. Simulation of the inner-loop of Subsystem 1.

\subsubsection{Outer-loop controller}

In the outer-loop of Subsystem 1, we use a P-controller $K_{P_{1}}$ (Fig. 7). We can redraw this system as shown in Fig. 8, in which $G_{i n_{1}}=\frac{1}{S} C_{1}\left(S I-\left(A_{1}+B_{1} F_{1}\right)\right)^{-1} B_{1} G_{1}$. It can be shown that $G_{i n_{1}}$ is a $2 \times 2$ multi-variable system. Unfortunately, in general, designing a P-controller for an MIMO system is difficult. However, if we consider $K_{p_{1}}$ in diagonal form as $K_{p_{1}}=k_{p_{1}} I_{2 \times 2}$, we can apply the generalized Nyquist theorem (Postlethwaite \& MacFarlane, 1979) to design $k_{p_{1}}$ such that it stabilizes the system as described in the following part.

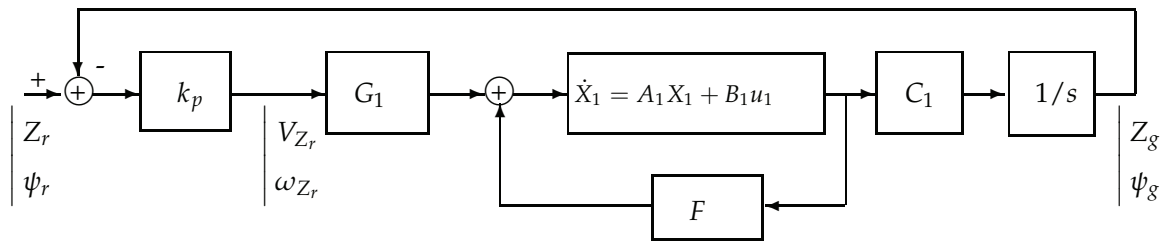

Fig. 7. Control structure of Subsystem 1.

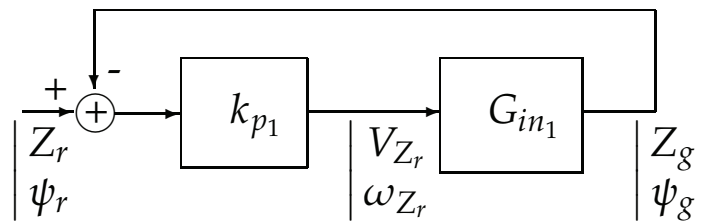

Fig. 8. Redrawing the control structure of Subsystem 1.

\subsubsection{Stability analysis}

The characteristic loci of $G_{i n_{1}}$ are shown in Fig. 9, where the dash-dot lines correspond to the infinite values. In Subsystem 1, Fig. 8, the inner-loop has already been stabilized, using an $H_{\infty}$ 
controller. Therefore, due to the presence of the integral term, $G_{i n_{1}}$ has two poles at the origin and the remaining poles are in the LHP plane. Hence, $G_{i n_{1}}$ has no pole in the Nyquist contour. It follows from the form of the characteristic loci of $G_{i n_{1}}$ in Fig. 9, that $k_{p_{1}} \in(0, \infty)$ will keep the entire system stable. However, in practice, we are subjected to the selection of small values of $k_{p_{1}}$ to avoid saturation of the actuators. $k_{p_{1}}=1.5$ is a typical value.

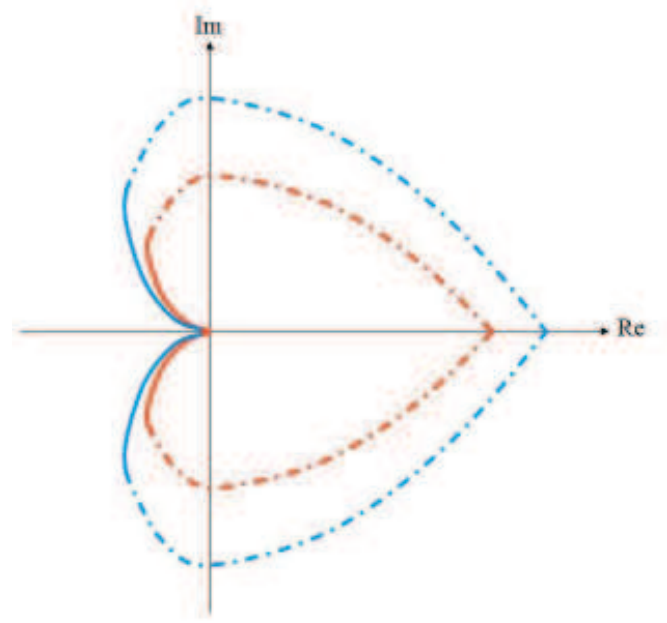

Fig. 9. Characteristic loci of $G_{i n_{1}}$.

\subsubsection{Tuning the controller}

With the above outer-loop controller, the stability of the whole system has been achieved; however, the controller in the form of $K_{p_{1}}=k_{p_{1}} \times I_{2 \times 2}$ with only one control parameter is not an appropriate choice. We need to have more degrees of freedom to tune the controller and achieve better performance. By considering the proportional feedback gain $K_{p_{1}}=$ $\operatorname{diag}\left\{K_{p_{11}}, K_{p_{12}}\right\}$ as a diagonal matrix, we have more degrees of freedom and can control each of the output channels in a decentralized manner, while keeping the system decoupled.

Uncertainty analysis usually is used to investigate the effect of the plant uncertainty. Here, we borrow this idea to analyze the effect of deviation of the diagonal entries of the matrix $K_{p_{1}}=k_{p_{1}} I_{2 \times 2}$ in the controller part. Alternatively, one can define $K_{p_{1}}$ as follows:

$$
K_{p_{1}}=\left[\begin{array}{cc}
K_{p_{11}} & 0 \\
0 & K_{P_{12}}
\end{array}\right]=k_{p_{1}} I_{2 \times 2}+\left[\begin{array}{cc}
\Delta_{K_{p_{11}}} & 0 \\
0 & \Delta_{K_{P_{12}}}
\end{array}\right]
$$

The objective is to design $\Delta=\operatorname{diag}\left\{\Delta_{K_{p_{11}}}, \Delta_{K_{p_{12}}}\right\}$ such that it does not affect the stability of the system. In fact, $\triangle$ is the tuning range (Fig. 10).

Following from Fig. 10, one can extract the internal model of the system as:

$$
\left\{\begin{array}{l}
y=G_{i n_{1}} k_{p_{1}}\left(I+G_{i n_{1}} k_{p_{1}}\right)^{-1} v+\left(I+G_{i n_{1}} k_{p_{1}}\right)^{-1} G_{i n_{1}} z \\
x=\left(I+G_{i n_{1}} k_{p_{1}}\right)^{-1} v-\left(I+G_{i n_{1}} k_{p_{1}}\right)^{-1} G_{i n_{1}} z
\end{array}\right.
$$

To simplify the notation, (20) can be rewritten as:

$$
\left\{\begin{array}{l}
y=G_{11} v+G_{12} z \\
x=G_{21} v+G_{22} z
\end{array}\right.
$$




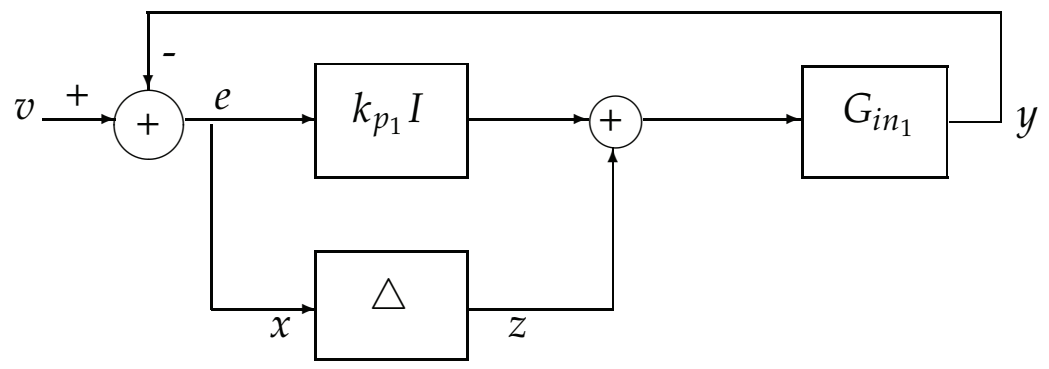

Fig. 10. Tuning the controller using uncertainty analysis.

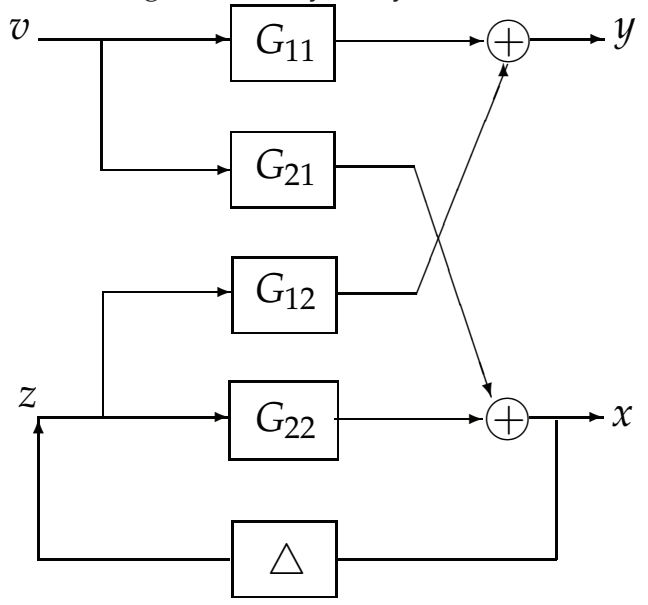

Fig. 11. Redrawing Subsystem 1 for uncertainty analysis.

Therefore, Fig. 10 can be redrawn as it is shown in Fig. 11. In the new diagram, since the nominal system with $\Delta=0$ is stable, all $G_{i j}$ are stable. $G_{11}, G_{12}$, and $G_{21}$ are outside the uncertain loop and cannot be affected by block $\triangle$; however, the loop includes $G_{22}$ and $\triangle$ may affect the internal stability of the system due to perturbations of the elements of $\triangle$. Since $G_{22}$ and $\triangle$ are stable, according to the generalized Nyquist theorem, the characteristic loci of the loop transfer function should not encircle the point $(-1+\mathrm{j} 0)$, or equivalently, $\left|\lambda_{i}\left(-G_{22} \Delta\right)\right|<1$. To satisfy this condition, since $\left|\lambda_{i}\left(G_{22} \Delta\right)\right| \leq \bar{\sigma}\left(G_{22} \Delta\right) \leq \sup _{\omega}\left(\bar{\sigma}\left(G_{22} \Delta\right)\right)=\left\|G_{22} \Delta\right\|_{\infty}$, it is sufficient that $\left\|G_{22} \Delta\right\|_{\infty}<1$. Using norm properties, we have:

$$
\left\|G_{22} \Delta\right\|_{\infty} \leq\left\|G_{22}\right\|_{\infty}\|\Delta\|_{\infty}
$$

Therefore, the sufficient condition for the stability of the system is:

$$
\left\|G_{22}\right\|_{\infty}\|\Delta\|_{\infty}<1
$$

For these values of the controller and plant and for a frequency range of $(0,10000)$, we obtain $\left\|G_{22}\right\|_{\infty}=0.6986$. Therefore, the perturbation of $K_{p_{1}}$ should be such that $\|\Delta\|_{\infty} \leq 1.4315$. Recall that $\triangle$ has a diagonal structure, and hence, all diagonal entries of $K_{p_{1}}$ should have less than a 1.4315-unit deviation from their nominal value. In fact, using this approach, we first 
obtained a nominal controller that provides the stability of the system, and then, we attempted to tune the controller to improve the performance, while keeping the system stable. After tuning the controller, the value of $K_{p_{1}}=\operatorname{diag}\{0.5,0.7\}$ was selected as an appropriate value that satisfies the above mentioned condition and gives a satisfactory performance. The method is conservative as $\Delta$ is structured and real, but applying to the UAV plant it has provided sufficient degree of freedom for tuning the controller and improving the performance.

To simulate the resulting system, let the outer-loop reference be $\left(Z_{r}, \psi_{r}\right)=(-2,0.5)$ and the current position and heading angle be $\left(Z_{g}, \psi_{g}\right)=(0,0)$. The system will reach its target after approximately $8 \mathrm{sec}$ as shown in Fig. 12.
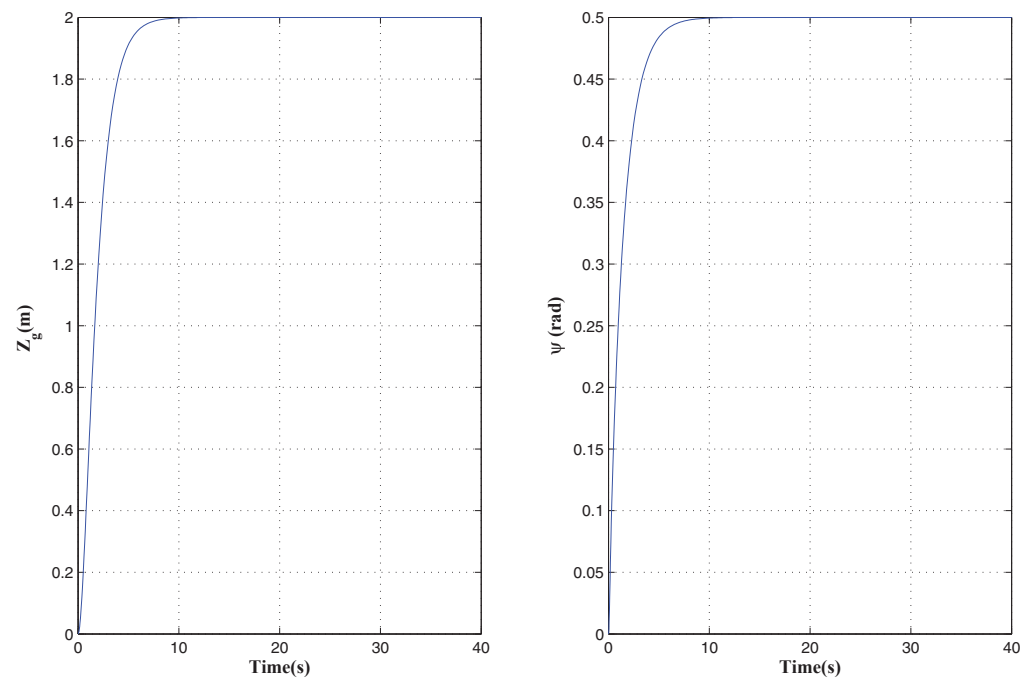

Fig. 12. Simulation of the outer-loop of Subsystem 1.

\subsection{Designing the controller for subsystem 2}

\subsubsection{Inner-loop controller}

For Subsystem 2, described by (11), we use an $H_{\infty}$ controller for the inner-loop controller design, similar to Subsystem 1. Analogous with Subsystem 1, we define $h_{2}$ as $h_{2}=C_{22} x_{2}+D_{22} u_{2}$, where

$$
C_{22}=\left[\begin{array}{cccccccc}
0.3162 & 0 & 0 & 0 & 0 & 0 & 0 & 0 \\
0 & 0.3162 & 0 & 0 & 0 & 0 & 0 & 0 \\
0 & 0 & 0.3162 & 0 & 0 & 0 & 0 & 0 \\
0 & 0 & 0 & 0.3162 & 0 & 0 & 0 & 0 \\
0 & 0 & 0 & 0 & 1 & 0 & 0 & 0 \\
0 & 0 & 0 & 0 & 0 & 1 & 0 & 0 \\
0 & 0 & 0 & 0 & 0 & 0 & 1 & 0 \\
0 & 0 & 0 & 0 & 0 & 0 & 0 & 1
\end{array}\right]
$$


$D_{22}=\left[\begin{array}{cc}5.4772 & 0 \\ 0 & 5.4772 \\ 0 & 0_{8 \times 2}\end{array}\right]$

With these parameters, we obtain $\gamma_{\infty}^{*}=0.0731$, and choosing $\gamma_{\infty}=0.0831$, we have:

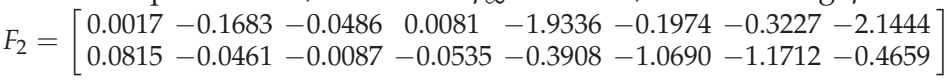

Moreover, $G_{2}=-\left(C_{2}\left(A_{2}+B_{2} F_{2}\right)^{-1} B_{2}\right)^{-1}$, is the feedforward gain for Subsystem 2 and can be calculated as:

$$
G_{2}=\left[\begin{array}{ll}
-0.0029 & 0.2335 \\
-0.0978 & 0.0632
\end{array}\right]
$$

The simulation of the system is shown in Fig. 13. In this figure, the initial state of the system is $x_{2}(0)=\left[\begin{array}{llllllll}1.5 & 0 & 0 & 0 & 0.17 & 0 & 0 & 0\end{array}\right]^{\prime}$. The injected disturbance has a maximum amplitude of 10 $\mathrm{m} / \mathrm{s}$ along the $x$ and $y$ axes (the $z$ direction does not affect the dynamics of Subsystem 2). The controlled system reaches the steady hovering state after $3.5 \mathrm{sec}$, and the disturbance effect is reduced to less than $0.25 \%$. In addition, the control inputs are within the unsaturated region.
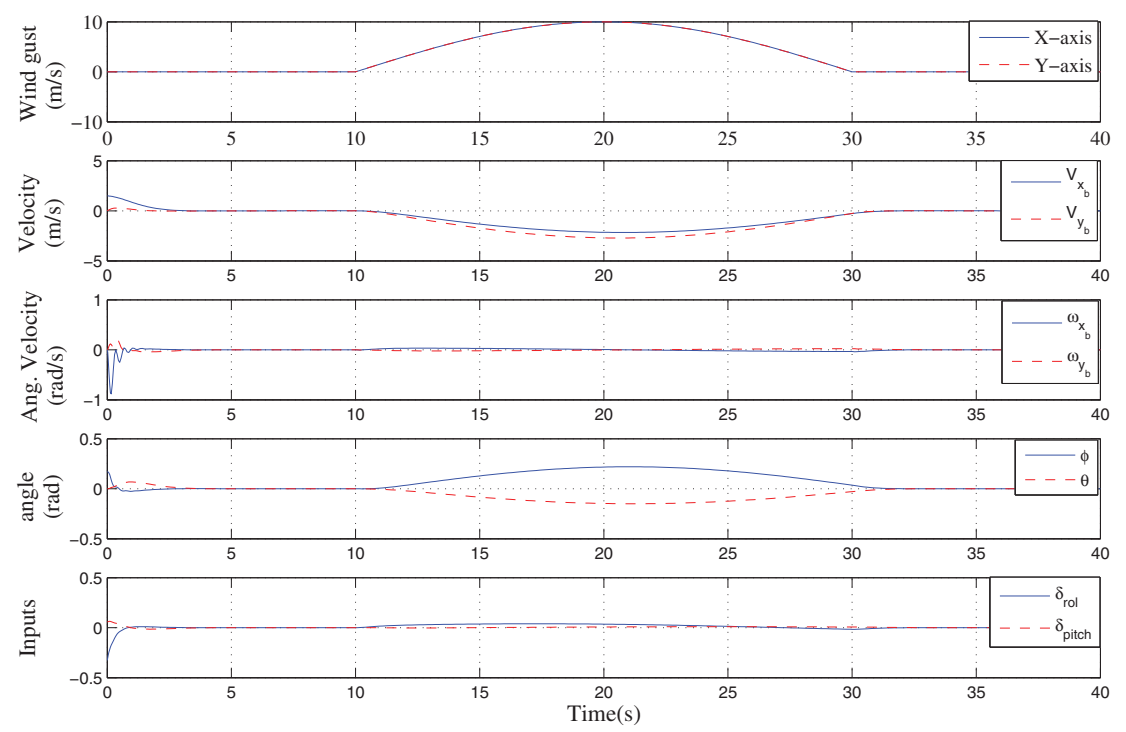

Fig. 13. Simulation of the inner-loop of Subsystem 2.

\subsubsection{Outer-loop controller}

Although the outer-loop of Subsystem 2 is similar to the outer-loop of Subsystem 1, the main difference lies in the presence of the nonlinear term, $R^{-1}$, in the outer-loop of Subsystem 2, as shown in Fig. 4. In this structure, it can be seen that the error signal is the difference between the actual position and the reference position, which both are in the ground frame. Therefore, the resulting control signal, which is the reference for the inner-loop, will be obtained in the ground frame; however, the inner-loop is in the body frame. Hence, it is reasonable that 
we transform the control signal to the body frame before delivering it to the inner-loop as the reference to be tracked. To implement this idea, we can use the transformation term, $R$, to obtain a control signal in the body frame. The new structure is shown in Fig. 14, in which $G_{i n_{2}}=C_{2}\left(S I-\left(A_{2}+B_{2} F_{2}\right)\right)^{-1} B_{2} G_{2}$ is a $2 \times 2$ multi-variable system. In Fig. 15, it is shown that the inner-loop block $G_{i n_{2}}$ is very close to a decoupled system with equal diagonal elements. Indeed, Subsystem 2 corresponds to the dynamics of the helicopter for the $x-y$ plane movement. In practice, we expect the dynamics of the UAV in the $x$ and $y$ directions to be similar and decoupled, since the pilot can easily drive the UAV in either of directions independently. Using this concept, we can take the block $G_{i n_{2}}$ out so that the two rotation matrices $R$ and $R^{-1}$ will cancel each other.

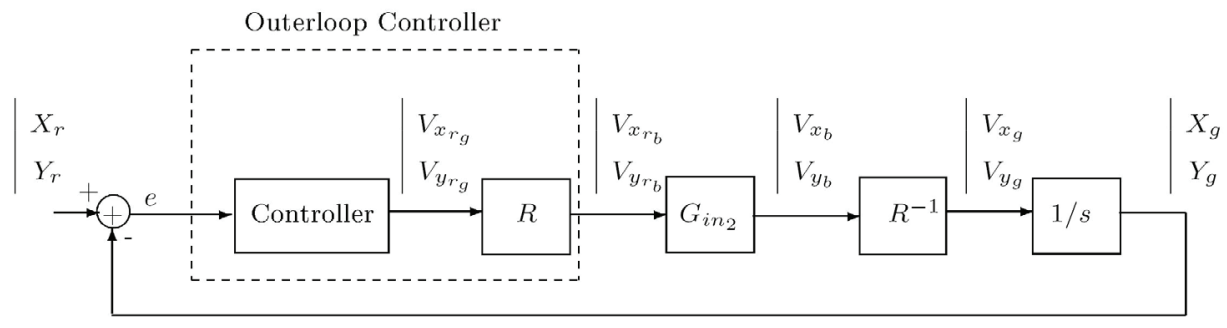

Fig. 14. Control diagram of Subsystem 2.
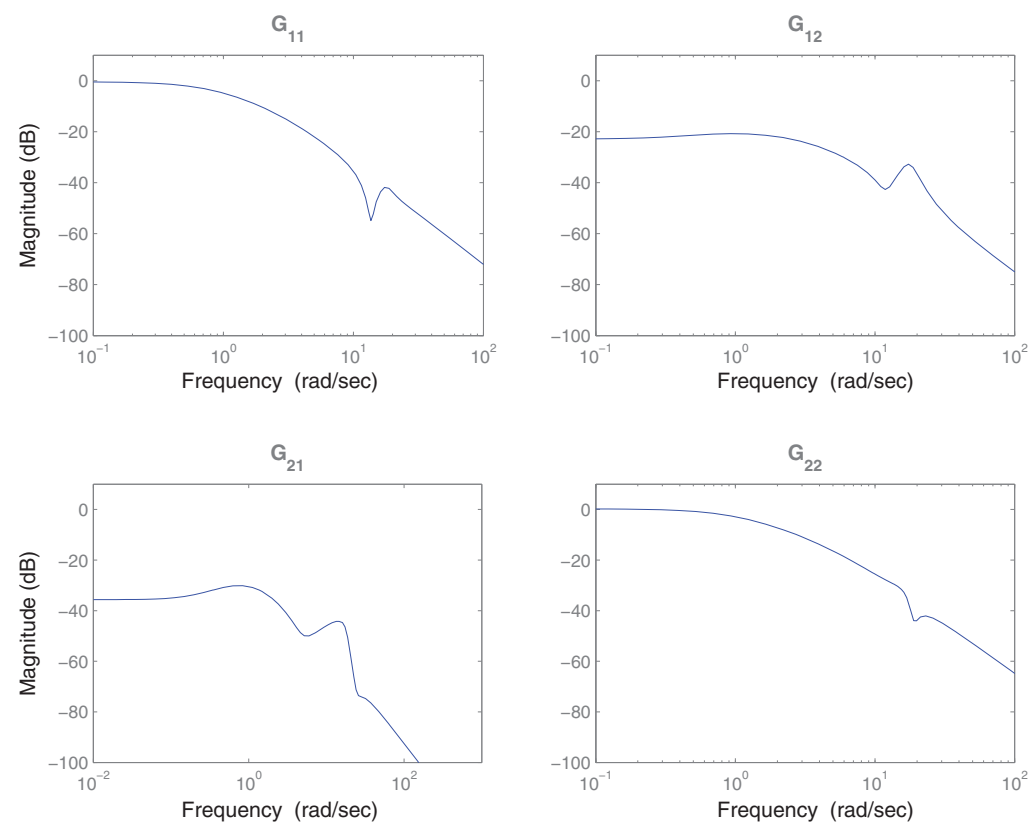

Fig. 15. Bode plot of entries of $G_{i n_{2}}$ 
The remaining job is simple, and we can repeat the procedure of designing the outer-loop controller for Subsystem 1 and design a P-controller in the form of $K_{p_{2}}=\operatorname{diag}\left\{K_{p_{21}}, K_{P_{22}}\right\}$ that stabilizes $G_{i n_{2}}$ (Fig. 16). As an appropriate choice of control parameters, we can select $K_{p_{2}}=\operatorname{diag}\{0.3,0.3\}$. Rationally, $K_{p_{21}}$ and $K_{P_{22}}$ should be the same, since we expect a similar behavior of the UAV system in the $x$ and $y$ directions.

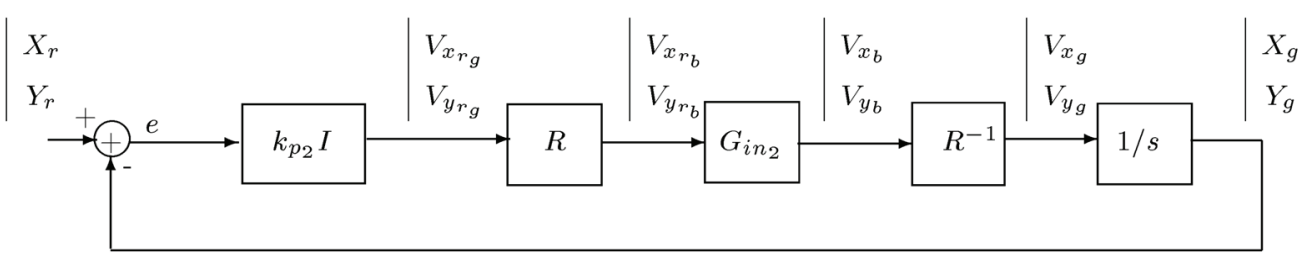

Fig. 16. Redrawing the control diagram of Subsystem 2.

For an outer-loop reference at $\left(x_{r}, y_{r}\right)=(2,2)$ and the current UAV position at $\left(x_{g}, y_{g}\right)=$ $(0,0)$, the simulation results are shown in Fig. 17, in which the UAV reaches to the desired position after approximately $10 \mathrm{sec}$, smoothly and without overshooting.
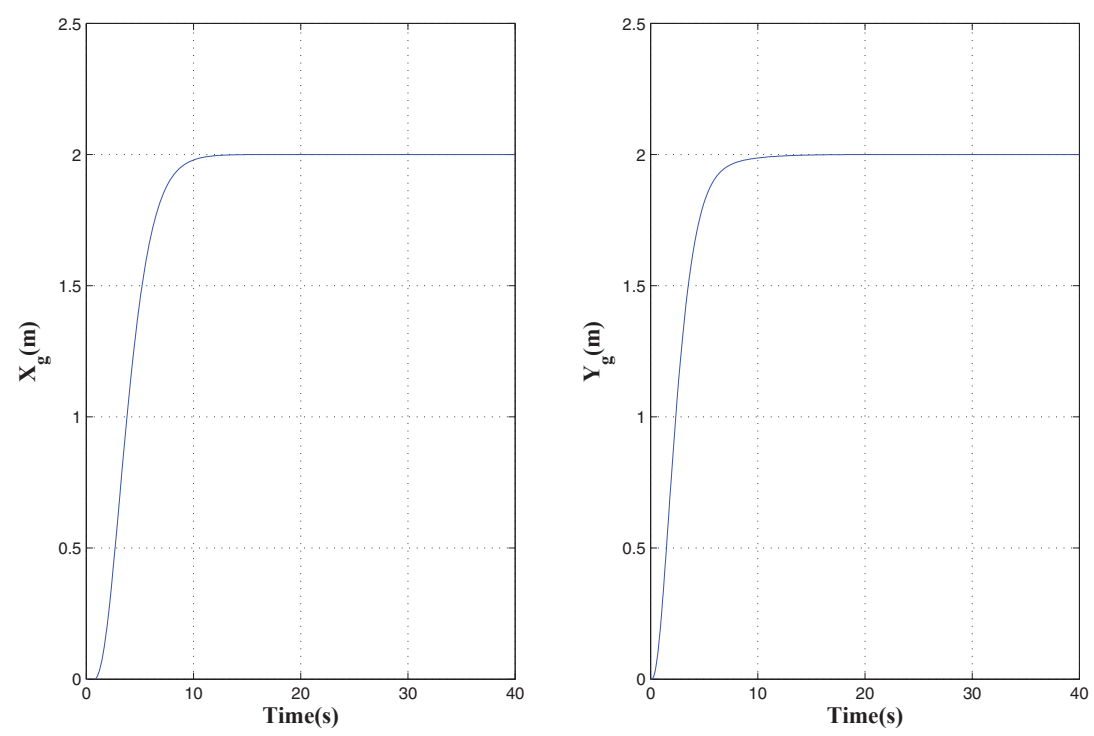

Fig. 17. Simulation of the outer-loop of Subsystem 2.

\section{Experimental results}

Before using the designed controller in an actual flight test, we first evaluate it through a hardware-in-the-loop simulation platform (Cai et al., 2009). In this platform, the nonlinear dynamics of the UAV has been replaced with its nonlinear model, and all software and hardware components that are involved in a real flight test remain active during the 
simulation. Using the hardware-in-the-loop simulation environment, the behavior of the system is very close to the real experiments.

Then, we conducted actual flight tests to observe the in-flight behavior of the helicopter. First, we used the UAV in the hovering state for $80 \mathrm{sec}$. Figure 18 shows the state variables in the hovering experiment at $(x, y, z, \psi)=(-16,-34,10,-1.5)$. To evaluate the hovering control performance, the position of the UAV is depicted in a 2D $x-y$ plane in Fig. 19. As can be seen, the position of the UAV has at most a 1-meter deviation from the desired hovering position, which is quite satisfactory. The control inputs are also shown in Fig. 20. All of the control inputs are within the unsaturated region.

Next, we used the UAV to follow a circle with a diameter of 20 meters as a given trajectory. This trajectory determines the reference $(x(t), y(t), z(t), \psi(t))$ for the system. With this trajectory, the UAV should complete the circle within $63 \mathrm{sec}$, while keeping a fixed altitude. Then, it will hover for 7 sec. In Fig. 21, it is shown that the UAV is able to follow this trajectory successfully. The UAV path tracking in the $x-y$ plane is shown in Fig. 22. Moreover, to have a better insight of the system behavior, all of the states of the UAV and the control inputs are represented in Fig. 23 and Fig. 24, respectively. These results show that the UAV is able to track the desired trajectory in situations close to the hovering state. The small deviations in the hovering mode or path tracking mode could be due to environmental effects such as wind disturbances or the GPS signal inaccuracy as with the installed sensors the measurable steady accuracy of the heading angle is $2.5^{\circ}$ and the positioning accuracy of the GPS is $3 m(1 \sigma)$. Videos of the hovering experiment and circle path tracking are available at http://uav.ece.nus.edu.sg/video/hover.mpg and http://uav.ece.nus.edu.sg/video/circle.mpg, respectively.
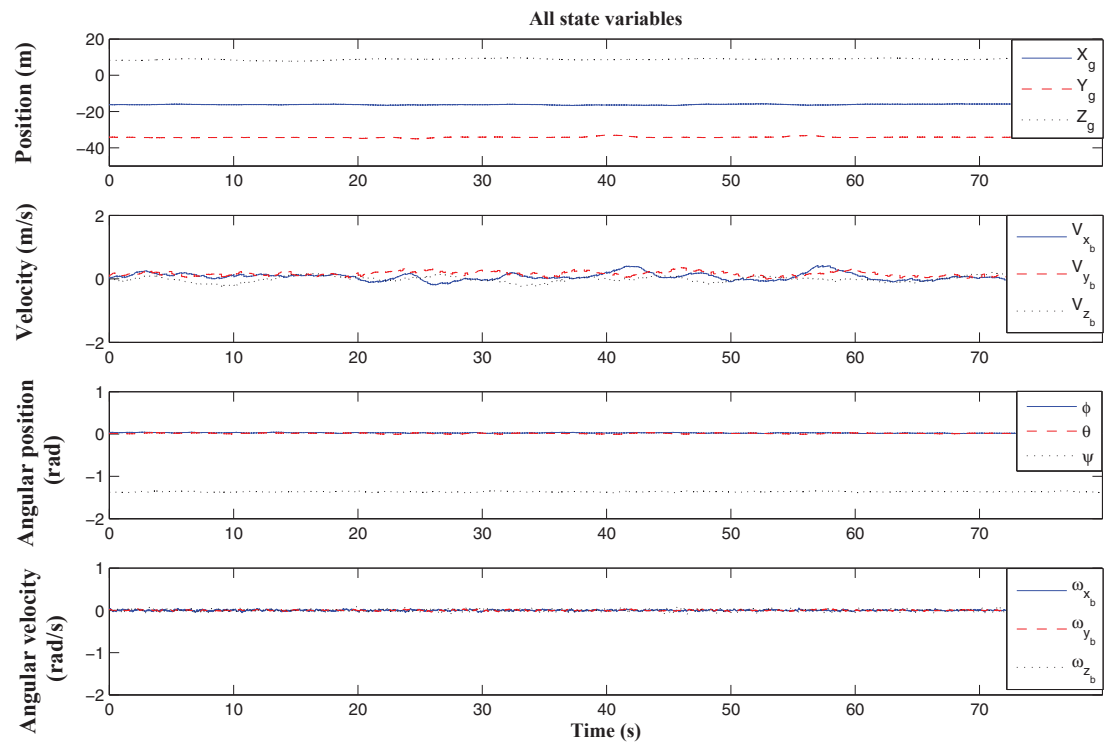

Fig. 18. State variables of the UAV for the hovering. 


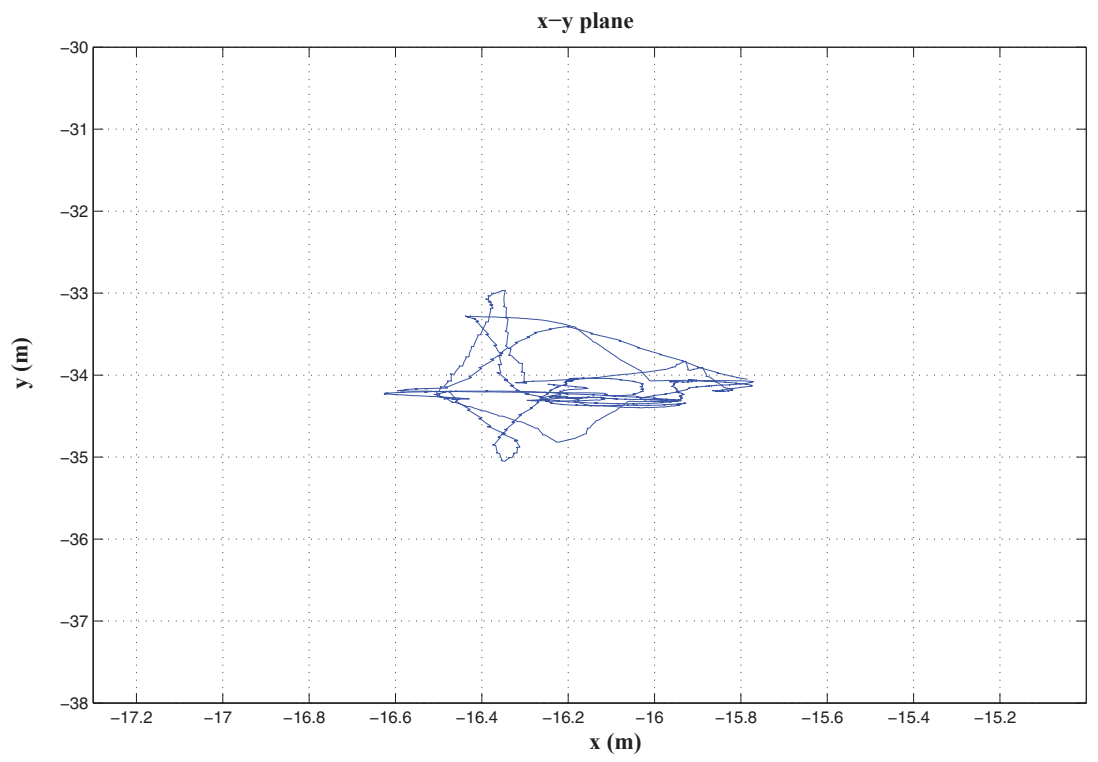

Fig. 19. UAV position in $x-y$ plane at hovering

\section{Conclusion}

In this chapter, we presented a systematic approach for the flight control design of a small-scale UAV helicopter in a hierarchical manner. In this structure, the lower level aims at stabilization of the system, and the upper level focuses on the reference tracking. For the disturbance attenuation and stabilization of the UAV, we used an $H_{\infty}$ controller in the inner-loop of the system. Due to the presence of some nonlinear terms in the outer-loop of the system, we first compensated for the nonlinearity by an inverse rotation; then, we used a decentralized P-controller to enable the UAV to follow a desired trajectory. We also proposed a new method of designing a P-controller for MIMO systems that was successfully applied to the UAV system. The simulations and actual flight tests show the efficacy of the control structure. In the future, we will use this structure to accomplish more complex missions such as formation control (Karimoddini et al., 2010). Such missions will require an embedded decision-making unit to support the tasks and to switch between the controllers. This concept will guide us in designing a hybrid supervisory controller in the path planner level of the UAV to comprehensively analyze the reactions between the continuous dynamics of the system and discrete switching between the controllers (Karimoddini et al., 2009).

\section{Acknowledgments}

The authors gratefully acknowledge the technical support of Mr. Dong Xiangxu and Mr. Lin Feng during the implementations and flight tests. 

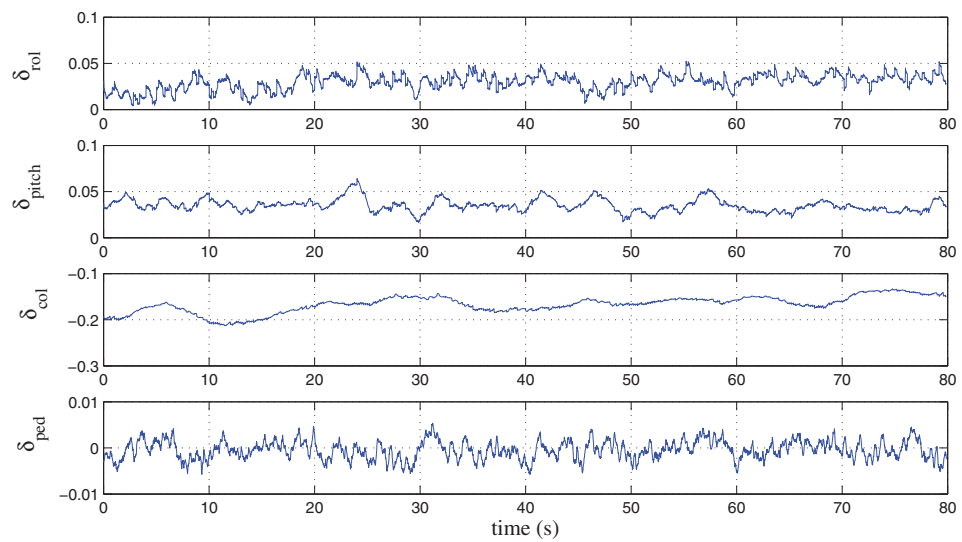

Fig. 20. Control signals at hovering
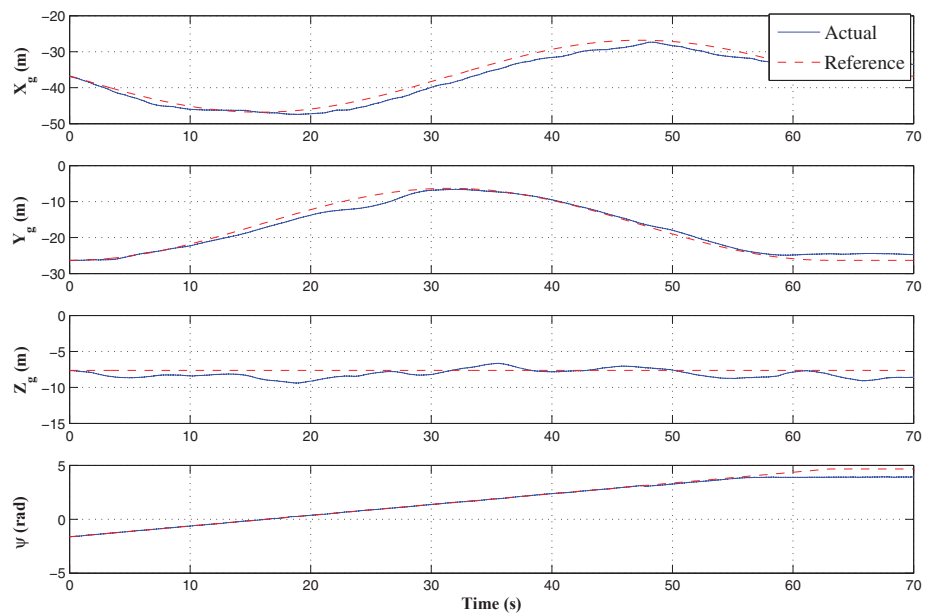

Fig. 21. Tracking a desired path. 


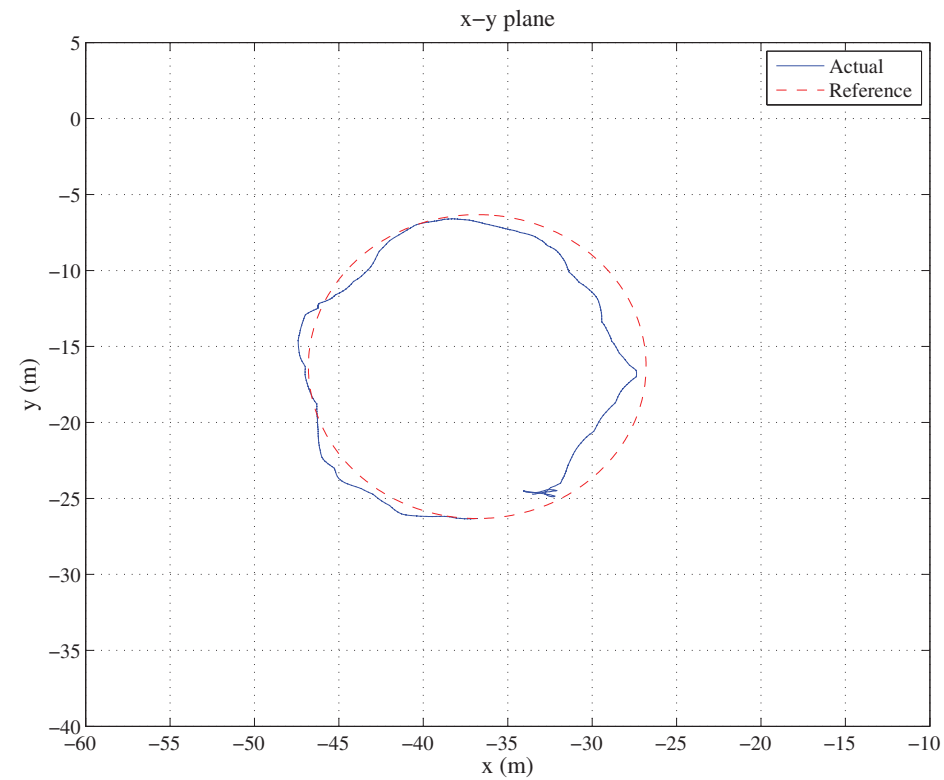

Fig. 22. Circle path tracking in $x-y$ plane.
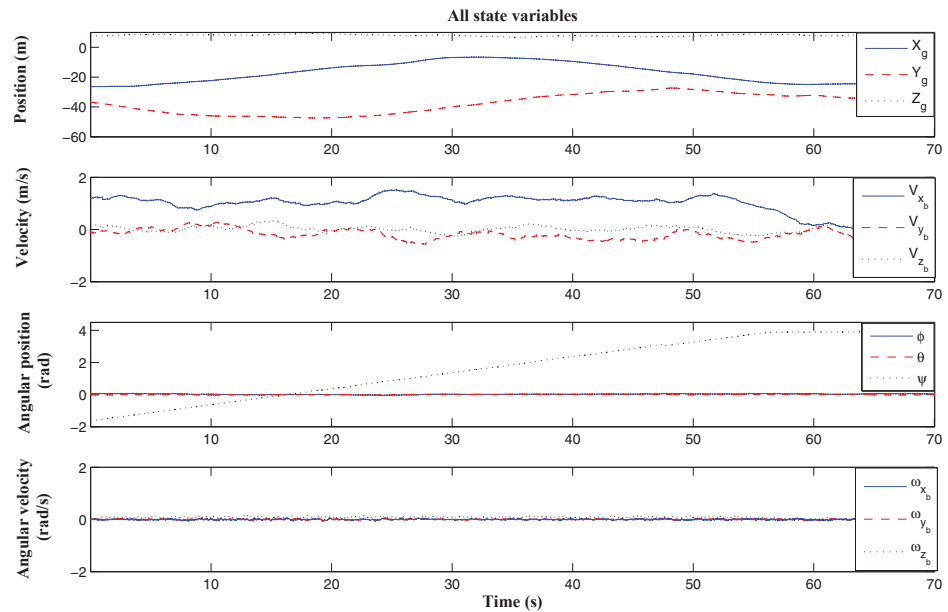

Fig. 23. States of the UAV in the circle path tracking behavior. 


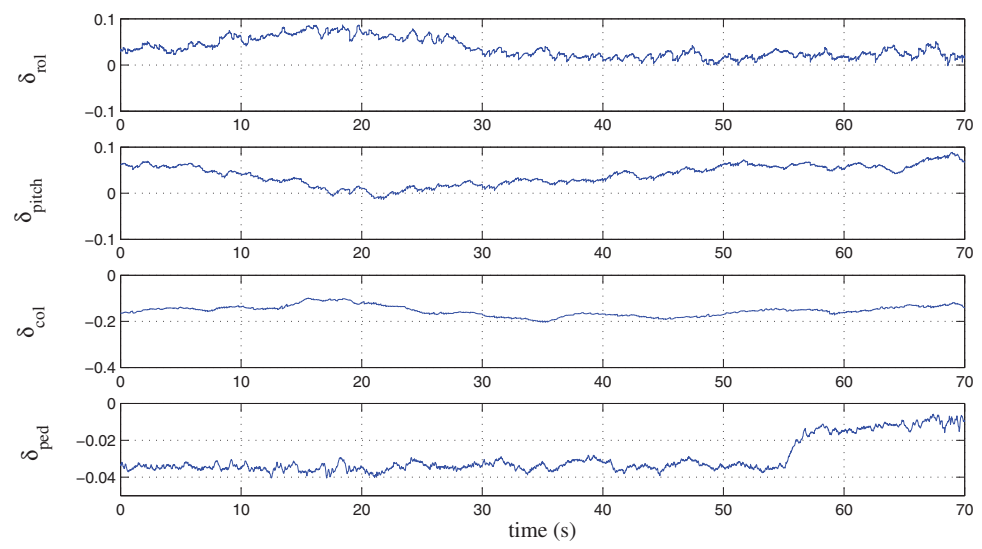

Fig. 24. Control inputs in the circle path tracking behavior.

\section{Nomenclature}

$A \quad=$ system matrix

$a_{s} \quad=$ longitudinal blade angle

$B=$ control input matrix

$B_{b} \quad=$ transformation matrix from the ground to the body frame

$b_{s} \quad=$ lateral blade angle

$E \quad=$ disturbance matrix

$\vec{F} \quad=$ resultant force in the body frame

$\vec{g} \quad=$ acceleration due to gravity, $\vec{g}=[0,0, g]^{\prime}$

$J \quad=$ inertia matrix

$\vec{M} \quad$ = resultant moment in the body frame

$\vec{P} \quad=$ position of the UAV, $\vec{P}=(X, Y, Z)^{\prime}$

$u \quad=$ control input vector

$\vec{V} \quad=$ linear velocity vector of the $\mathrm{UAV}, \vec{V}=\left(V_{x}, V_{y}, V_{z}\right)^{\prime}$

$\vec{\omega} \quad=$ angular velocity vector of the UAV, $\vec{\omega}=\left(\omega_{x}, \omega_{y}, \omega_{z}\right)^{\prime}$

$w \quad=$ wind gust disturbance in the body frame, $w=\left(w_{x}, w_{y}, w_{z}\right)^{\prime}$

$\theta \quad=$ pitch angle

$\phi \quad=$ roll angle

$\psi \quad=$ yaw angle

$\delta_{\text {roll }}=$ roll channel input

$\delta_{\text {pitch }}=$ pitch channel input

$\delta_{\text {pedal }}=$ pedal channel input

$\delta_{\text {col }}=$ collective channel input

$\star_{g}=$ the value of $\star$ in the ground frame

$\star_{b}=$ the value of $\star$ in the body frame

$\star_{r}=$ the value of $\star$ as control reference 


\section{References}

Bortoff, S. (1999). The university of toronto rc helicopter: a test bed for nonlinear control, Proceedings of the IEEE International Conference on Control Applications, IEEE, Vol. 1, pp. 333-338.

Cai, G., Peng, K., Chen, B. M. \& Lee, T. H. (2005). Design and assembling of a uav helicopter system, International Conference on Control and Automation, Vol. 2, pp. 697-702.

Cai, G., Chen, B. M., Peng, K., Dong, M. \& Lee, T. H. (2006). Modeling and control system design for a uav helicopter, 14th Mediterranean Conference on Control and Automation, pp. 1-6.

Cai, G., Feng, L., Chen, B. M. \& Lee, T. H. (2008a). Systematic design methodology and construction of uav helicopters, Mechatronics, 18(10): 545-558.

Cai, G., Chen, B. M., Peng, K., Dong, M. \& Lee, T. H.(2008b). Comprehensive modeling and control of the yaw channel of a UAV helicopter, IEEE Transactions on Industrial Electronics, 55(9): 3426-3434.

Cai, G., Chen, B. M., Lee, T. H. \& Dong, M. (2009). Design and implementation of a hardware-in-the-loop simulation system for small-scale UAV helicopters, Mechatronics, 19(7): 1057-1066.

Campbell, M. E. \& Wheeler, M. (2010). Vision-Based Geolocation Tracking System for Uninhabited Aerial Vehicles, AIAA Journal of Guidance, Control, and Dynamics, 33(2): 521-532.

Chen, B. M. (2000). Robust and $H_{\infty}$ Control, Springer, London.

Dong, M., Chen, B. M., Cai, G. \& Peng, K. (2007). Development of a real-time onboard and ground station software system for a UAV helicopter, AIAA Journal of Aerospace Computing, Information, and Communication, 4(8): 933-955.

Enns, R. \& Si, J. (2003). Helicopter trimming and tracking control using direct neural dynamic programming, IEEE Transactions on Neural Networks, IEEE, Vol. 14, pp. 929-939, 2003.

Gavrilets, V., Shterenberg, A., Dahleh, M. \& Feron, E. (2000). Avionics system for a small unmanned helicopter performing aggressive maneuvers, Proceedings of the 19th Digital Avionics Systems Conferences, Vol. 1, pp. 1E2/1-1E2/7.

Isidori, A., Marconi, L. \& Serrani, A.(2003) Robust nonlinear motion control of a helicopter, IEEE Transactions on Automatic Control, IEEE, Vol. 48, pp. 413-426, 2003.

Karimoddini, A., Lin, H., Chen, B.M. \& Lee, T.H. (2009) Developments in hybrid modeling and control of unmanned aerial vehicles, Proceedings of the 7th IEEE International Conference on Control and Automation, Christchurch, New Zealand, pp. 228-233.

Karimoddini, A., Lin, H., Chen, B.M. \& Lee, T.H. (2010) Hybrid formation control of the Unmanned Aerial Vehicles, Mechatronics, doi:10.1016/ j.mechatronics.2010.09.007.

Kim, J. \& Sukkarieh, S. (2007). Real-time implementation of airborne inertial-SLAM, Journal of Robotics and Autonomous Systems, 55(1): 62-71.

Kuroki, Y., Young, G.S. \& Haupt, S.E. (2010). UAV navigation by an expert system for contaminant mapping with a genetic algorithm, Journal of Expert Systems with Applications, 37(6): 4687-4697.

Metni, N. \& Hamel, T. (2007). A UAV for bridge inspection: Visual servoing control law with orientation limits, Journal of Automation in Construction, 17(1): 3-10.

Peng, K., Dong, M., Chen, B. M., Cai, G., Lum, K. Y. \& Lee, T. H. (2007). Design and implementation of a fully autonomous flight control system for a uav helicopter, Chinese Control Conference, pp. 662-667. 
Peng, K. , Cai, G., Chen, B. M., Dong, M., Lum, K. Y. \& Lee, T. H. (2009). Design and implementation of an autonomous flight control law for a UAV helicopter, Automatica, 45(10), pp. 2333-2338.

Postlethwaite, I. \& MacFarlane, A. G. I. (1979). A Complex Variable Approach to the Analysis of Linear Multivariable Feedback Systems, in Lecture Notes in Control and Information Sciences, Vol. 12/1979, Springer, Berlin, pp. 58-76.

Saripalli, S., Montgomery, J. \& Sukhatme, G. (2003). Visually guided landing of an unmanned aerial vehicle, IEEE Transactions on Robotics and Automation, 19(3): 371-380.

Shaferman, V. \& Shima, T. (2008). Unmanned Aerial Vehicles Cooperative Tracking of Moving Ground Target in Urban Environments, AIAA Journal of Guidance, Control, and Dynamics, 31(5): 1360-1371.

Shim, D. H., Kim, H. J. \& Sastry, S. (2003). Decentralized nonlinear model predictive control of multiple flying robots, Proceedings of the 42nd IEEE Conference on Decision and Control, IEEE, Hawaii, pp. 3621-3626.

Stevens, B. L. \& Lewis, F. L. (1992). Aircraft control and simulation, Wiley, New York.

Wang, Q.G., Lin, C., Ye, Z., Wen, G., He, Y. \& Hang, C. C. (2007). A quasi-lmi approach to computing stabilizing parameter ranges of multi-loop pid controllers, Journal of Process Control, 17(1): 59-72. 


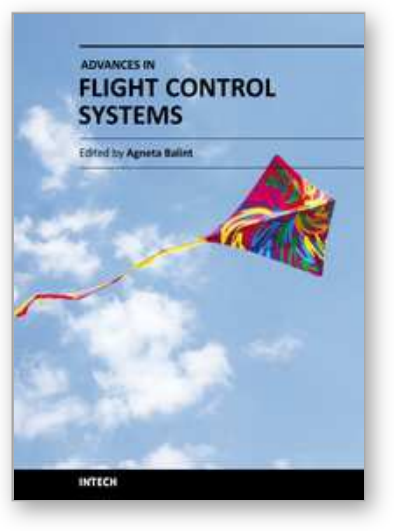

\author{
Advances in Flight Control Systems \\ Edited by Dr. Agneta Balint
}

ISBN 978-953-307-218-0

Hard cover, 296 pages

Publisher InTech

Published online 11, April, 2011

Published in print edition April, 2011

Nonlinear problems in flight control have stimulated cooperation among engineers and scientists from a range of disciplines. Developments in computer technology allowed for numerical solutions of nonlinear control problems, while industrial recognition and applications of nonlinear mathematical models in solving technological problems is increasing. The aim of the book Advances in Flight Control Systems is to bring together reputable researchers from different countries in order to provide a comprehensive coverage of advanced and modern topics in flight control not yet reflected by other books. This product comprises 14 contributions submitted by 38 authors from 11 different countries and areas. It covers most of the currents main streams of flight control researches, ranging from adaptive flight control mechanism, fault tolerant flight control, acceleration based flight control, helicopter flight control, comparison of flight control systems and fundamentals. According to these themes the contributions are grouped in six categories, corresponding to six parts of the book.

\title{
How to reference
}

In order to correctly reference this scholarly work, feel free to copy and paste the following:

Ali Karimoddini, Guowei Cai, Ben M. Chen, Hai Lin and Tong H. Lee (2011). Hierarchical Control Design of a UAV Helicopter, Advances in Flight Control Systems, Dr. Agneta Balint (Ed.), ISBN: 978-953-307-218-0, InTech, Available from: http://www.intechopen.com/books/advances-in-flight-control-systems/hierarchicalcontrol-design-of-a-uav-helicopter

\section{INTECH}

open science | open minds

\section{InTech Europe}

University Campus STeP Ri

Slavka Krautzeka 83/A

51000 Rijeka, Croatia

Phone: +385 (51) 770447

Fax: +385 (51) 686166

www.intechopen.com

\section{InTech China}

Unit 405, Office Block, Hotel Equatorial Shanghai

No.65, Yan An Road (West), Shanghai, 200040, China

中国上海市延安西路65号上海国际贵都大饭店办公楼 405 单元

Phone: +86-21-62489820

Fax: $+86-21-62489821$ 
(C) 2011 The Author(s). Licensee IntechOpen. This chapter is distributed under the terms of the Creative Commons Attribution-NonCommercialShareAlike-3.0 License, which permits use, distribution and reproduction for non-commercial purposes, provided the original is properly cited and derivative works building on this content are distributed under the same license. 